\title{
BMJ Open Prevalence and correlates of pre- diabetes in adults of mixed ethnicities in the South African population: a systematic review and meta-analysis protocol
}

\author{
Aubrey Mbulelo Sosibo (D), Nomusa Christina Mzimela (D), \\ Phikelelani Siphosethu Ngubane, Andile Khathi
}

To cite: Sosibo AM,

Mzimela NC, Ngubane PS, et al. Prevalence and correlates of pre-diabetes in adults of mixed ethnicities in the South African population: a systematic review and meta-analysis protocol. BMJ Open 2021;11:e048266. doi:10.1136/ bmjopen-2020-048266

\section{- Prepublication history and} additional supplemental material for this paper are available online. To view these files, please visit the journal online (http://dx.doi.org/10.1136/ bmjopen-2020-048266).

AMS and AK are joint senior authors.

Received 21 December 2020 Accepted 22 September 2021

A Check for updates

(C) Author(s) (or their employer(s)) 2021. Re-use permitted under CC BY-NC. No commercial re-use. See rights and permissions. Published by BMJ.

Human Physiology, University of KwaZulu-Natal College of Health Sciences, Durban, KwaZuluNatal, South Africa

Correspondence to Aubrey Mbulelo Sosibo; sosibo.aubrey3@gmail.com

\section{ABSTRACT}

Introduction Pre-diabetes is a metabolic condition characterised by moderate glycaemic dysregulation and is a front-line risk factor to multiple metabolic complications such as overt diabetes. To the best of our knowledge, this will be the first systematic review and meta-analysis that focuses on generating a comprehensive pooling of studies that report on the pre-diabetes prevalence in South Africa. Therefore, the review's purpose will be to screen and elect reports that can be used to synthesise and provide the best estimate prevalence and correlate of pre-diabetes in the South African population.

Methods and analysis To determine the prevalence and correlates of pre-diabetes in South African, we will search PubMed, Embase and African Journal online for published or unpublished studies reporting the prevalence of prediabetes in South Africa starting from the year 2000 to 2020. Studies will be assessed for eligibility by checking if they meet the inclusion criteria. Eligible studies will undergo data extraction and risk of bias assessment. We will perform a subgroup analysis to detect probable causes of heterogeneity.

Ethics and dissemination The review will not require ethics clearance because non-identifiable data will be used. The review outcomes will give more insight into the current burden that pre-diabetes has in South Africa. PROSPERO registration number CRD42020182430.

\section{INTRODUCTION}

Pre-diabetes (or intermediate hyperglycaemia) is a serious health condition characterised by blood glucose concentration that is higher than normal but not high enough to diagnose with overt type 2 diabetes (T2D). ${ }^{12}$ The onset of pre-diabetes is preceded by moderate insulin resistance or pancreatic $\beta$ cell dysfunction, which, in turn, causes a gradual increase in glucose levels. ${ }^{13}$ The diagnosis of pre-diabetes is confirmed in individuals with impaired fasting glucose (IFG) or impaired glucose tolerance (IGT) or elevated glycosylated haemoglobin (HbA1c) using
Strengths and limitations of this study

- This will be the first comprehensive systematic review and meta-analysis based on the primary outcome of determining the total pre-diabetes prevalence in South Africa.

- The appraisal for the quality of study and strength of evidence will be done using the established Grading of Recommendations Assessment, Development and Evaluation method.

- Since the criteria for diagnosing pre-diabetes differs, the heterogeneity may be affected by the prediabetes diagnostic criteria utilised by each eligible study.

either WHO or American Diabetes Association (ADA). ${ }^{4}$ Therefore, when measuring pre-diabetes prevalence, the WHO and ADA criteria are generally used.

The International Diabetes Federation (IDF) estimated that the 2019 pre-diabetes global prevalence was $7.5 \%$ (373.9 million people). By 2045, the projected prevalence will increase to $8.6 \%$ (548.4 million). ${ }^{5}$ In all the seven regions of the IDF, predictions state that the African region will have the most significant increase in pre-diabetes prevalence. The projection is that the 2019 prevalence of 45.3 million will rise by $143.3 \%$ to 110.2 million. ${ }^{5}$ However, studies conducted in populations with various ethnic groups reported that pre-diabetes prevalence in certain ethnic groups is disproportionally high. ${ }^{6}$ In the UK, the observations showed that minority ethnic groups have a higher prevalence of pre-diabetes. ${ }^{7}$ Furthermore, another report revealed that $15.3 \%$ of adults of the 88 million estimated to have pre-diabetes did not know, which indicates that most people remain with this condition undiagnosed. ${ }^{5}$ 
This is concerning because the pre-diabetes condition can be very harmful.

Researchers have shown that pre-diabetes can be a toxic environment that instigates multiple metabolic complications, such as T2D. ${ }^{1}$ The literature indicates that pre-diabetes promotes severe chronic microvascular and macrovascular complications, blood pressure changes, fatty liver disease conditions and T2D. ${ }^{3}{ }^{4}$ A prevention study spanning over 20 years displayed a greater than $90 \%$ cumulative incidence of progression to T2D from IGT. ${ }^{8}$ This development rate is a concern because we know that diabetes causes the death of approximately 1.3 million people annually. ${ }^{9}$ In addition, the literature reveals that there is an increased risk of heart failure and all-cause mortality in people with pre-diabetes. ${ }^{10} 11$ Therefore, the synthesis of available prevalence data for pre-diabetes in a population may prove crucial in many ways.

The pooling of studies with pre-diabetes prevalence data will help determine the current burden that pre-diabetes has on a specific community and assist in mitigating the future prevalence of pre-diabetes and its associated metabolic complications. Subsequently, the findings have the potential to appraise social and healthcare professionals better. Notably, the prevalence of pre-diabetes is shown to vary among different populations. ${ }^{5}$ Therefore, populations must have their own summarised prevalence data so that relevant and empirical evidence can be at the policymakers' disposal, which will result in better-informed decisions.

Hence, the review will focus on the population in South Africa, which could also be a representative of Southern Africa. Various studies have reported on the prevalence of pre-diabetes in South Africa. ${ }^{12-14}$ However, non of the studies can independently give us an overall prevalence of pre-diabetes and account for all the ethnic groups. Moreover, there is no systematic review that focuses on the primary outcome of determining the total pre-diabetes prevalence in South Africa. Therefore, this led us to generate the following research questions and objectives.

\section{RESEARCH QUESTIONS}

1. What is the overall prevalence of pre-diabetes, and is there a fair representation of all the ethnic groups living in South Africa?

2. What are the common correlates of pre-diabetes?

\section{OBJECTIVES}

1. To determine the prevalence of pre-diabetes in the adult population of mixed ethnicities.

2. To determine the common correlates of pre-diabetes.

\section{METHODS AND ANALYSIS}

\section{Study design}

This systemic review protocol has been prepared following the Preferred Reporting Items for Systematic Reviews and Meta-Analyses Protocols 2015 guidelines.

\section{Search strategy}

With a librarian's aid, two independent reviewers will conduct a comprehensive search of databases to find all related articles published on diabetes mellitus and prediabetes in South Africa from January 2000 to May 2020 regardless of the language of publication. The databases that will be screened will include MEDLINE through PubMed, Google Scholar, Embase and African Journals Online. They will use some of the following Medical Subject Heading in our search strategy: "Pre-diabetes," "South Africa," "Prevalence," "Type 2 diabetes mellitus," "Impaired fasting glucose," and "Impaired glucose tolerance." A detailed method shown in online supplemental table 1 will be used to search PubMed. We will use the Mendeley referencing manager (V.1.19.10) to remove duplicates. Moreover, they will perform a hand searching to identify other eligible studies not indexed in the databases, especially in the included studies' bibliography and relevant literature reviews. We will also request and screen unpublished manuscripts and thesis from the University of KwaZulu-Natal registry and contact researchers.

\section{Types of study eligible}

The studies that we will consider for this review consist of prospective or retrospective cross-sectional population based and cohort studies reporting pre-diabetes prevalence in South Africa. The study must have a minimum of 100 participants for it to pass eligibility. There will be no language restriction for the eligibility of studies. Furthermore, the most up-to-date and comprehensive version will be selected for studies that will report the same results in multiple articles.

\section{Types of participants}

The participants in the studies included will need to be adults $(\geq 18)$ located in South Africa, registered citizens who are black, coloured, Indian/Asian or white. The participants used in the studies will need to be clinically diagnosed with pre-diabetes using the ADA or WHO diagnosis criteria. The diagnosis criteria defined by the ADA and the WHO will be considered. Accordingly, the diagnosis is determined by observing IFG, IGT and elevated HbA1c. ${ }^{15}$ IFG is defined as fasting plasma glucose of $5.6-6.9 \mathrm{mmol} / \mathrm{L}$. IGT is defined as the 2-hour plasma glucose of $7.8-11.0 \mathrm{mmol} / \mathrm{L}$ after a 2-hour interval following the ingestion of $75 \mathrm{~g}$ of an oral glucose load or a combination of both the IGT and the IFG recorded during the oral glucose tolerance test. Also, pre-diabetes diagnosis with $\mathrm{HbAlc}$ will be accepted for any value between $5.7 \%$ and $6.5 \% .^{15}{ }^{16}$ Any study lacking a clear diagnosis criteria description will be excluded if, after contacting authors twice, the information is not provided.

\section{Inclusion and exclusion criteria}

The two independent reviewers will be responsible for choosing eligible studies that meet the type of study and participant requirements. The articles or thesis included will have to be studies done between the years 2000 and 
2020. Therefore, all studies not adhering to the eligible type of study, participant requirements, and that fall outside the 20 year range between 2000 and 2020 will be excluded. Studies with insufficient data to calculate the primary outcome will face exclusion if the requested data is not provided after contacting the corresponding author twice.

\section{Primary outcomes}

- Prevalence of pre-diabetes.

- Prevalence of pre-diabetes across different ethnic groups.

\section{Secondary outcome}

- Determine the most conventional risk factors associated with pre-diabetes.

\section{Data extraction}

Using a predesigned excel form, the reviewer will extract the applicable data. To ensure the quality of extracted data, another reviewer will independently check all data. If there are any disagreements, they will be deliberated and solved with the assistance of a third reviewer. The data to be extracted will include the population sampled, crude pre-diabetes prevalence estimates, and any prevalence estimates reported stratified by age, sex or location (within South Africa). Data on parameters such as weight, hypertension and family history of diabetes will be pulled to appraise the most conventional risk factors associated with pre-diabetes. Prevalence figures and $95 \%$ CIs will be extracted or calculated, provided that all necessary data is made available. Where data are insufficient or presented graphically, we will contact the article's first author to request more data or calculate from the available data using Wilson's method. Given that all essential data will be provided, we will pull all correlates for pre-diabetes present in the clinically diagnosed participants.

\section{Assessment of the quality of included studies}

Two investigators (AMS and AK) will independently assess the methodological quality of comprised studies using the risk of bias tool for prevalence studies developed by Hoy and colleagues. ${ }^{17}$ A score of $0-4,5-7$ or $8-10$ rated the risk of bias as high, moderate or low, respectively. A third review author (NCM) will resolve disagreements between the two investigators by consensus or arbitration.

\section{Data synthesis and analysis}

The MetaXL (www.epigear.com) add-in for Microsoft Excel will be utilised for the synthesis and analysis. The point estimate for each study will be transformed with the Freeman-Tukey double arcsine method to stabilise the variance. The normal distribution of data will be validated using the D'Agostino \& Pearson omnibus normality test. The study-specific estimates with $95 \%$ CI will be pooled to generate an overall summary prevalence figure across the elected studies. After that, the heterogeneity between estimates will be assessed using the $\mathrm{I}^{2}$ statistics.
The $\mathrm{I}^{2}$ value describes the percentage of variation not because of chance or sampling error across studies. ${ }^{18}$ Suppose the $\mathrm{I}^{2}$ value is higher than $75 \%$. In that case, the heterogeneity between studies will be deemed high. ${ }^{19}$ Any possible influences on prevalence estimates will be investigated using subgroup analyses and meta-regression. Where studies allow, we will descriptively compare prevalence estimates by the following subgroups: sex, race, age, body mass index, lipid profile, family history, exercise and education. After that, we will calculate the regression coefficient to ascertain a linear relationship between the effect estimate (ie, outcome variable) and the explanatory variable or subgroup. We will then assess the influence on estimates of the following study-level variables identified a priori as potential sources of variation in the estimates of prevalence $^{1}$ : risk of bias, ${ }^{2}$ geographical location and ${ }^{3}$ data collection method. Finally, where fitting the outcomes will be displayed in tables or forest plots.

\section{Confidence in cumulative evidence}

The Grading of Recommendations Assessment, Development and Evaluation (GRADE) method will assess the strength of evidence. ${ }^{20}$ The GRADE method will provide a score of the quality of the studies and the strength of the evidence depending on methodological flaws within the included studies, consistency of results across diverse studies, precision estimates and publication bias.

\section{Patient and public involvement}

There was no patient and public involvement.

\section{ETHICS AND DISSEMINATION}

The systematic review and meta-analysis do not require ethics clearance since studies with non-identifiable data will be used. The review will give insight on the current burden that pre-diabetes has on specific areas in the country and may assist in predicting and mitigating future prevalence of other associated conditions like T2D.

\section{Twitter Nomusa Christina Mzimela @Chrinom}

Acknowledgements I want to thank the Rational Research Foundations and the College of Health Science for their financial support.

Contributors AMS, NCM and AK were responsible for brainstorming, designing the study and then also drafted the protocol. AMS, AK and PSN were responsible for reviewing and approving the final draft of the manuscript.

Funding The work is funded by the National Research Foundation (South Africa), grant number (121558).

Competing interests None declared.

Patient consent for publication Not applicable.

Provenance and peer review Not commissioned; externally peer reviewed.

Supplemental material This content has been supplied by the author(s). It has not been vetted by BMJ Publishing Group Limited (BMJ) and may not have been peer-reviewed. Any opinions or recommendations discussed are solely those of the author(s) and are not endorsed by BMJ. BMJ disclaims all liability and responsibility arising from any reliance placed on the content. Where the content includes any translated material, BMJ does not warrant the accuracy and reliability of the translations (including but not limited to local regulations, clinical guidelines, terminology, drug names and drug dosages), and is not responsible for any error and/or omissions arising from translation and adaptation or otherwise. 
Open access This is an open access article distributed in accordance with the Creative Commons Attribution Non Commercial (CC BY-NC 4.0) license, which permits others to distribute, remix, adapt, build upon this work non-commercially, and license their derivative works on different terms, provided the original work is properly cited, appropriate credit is given, any changes made indicated, and the use is non-commercial. See: http://creativecommons.org/licenses/by-nc/4.0/.

\section{ORCID iDs}

Aubrey Mbulelo Sosibo http://orcid.org/0000-0002-9617-5715

Nomusa Christina Mzimela http://orcid.org/0000-0001-6505-6708

\section{REFERENCES}

1 Tabák AG, Herder C, Rathmann W, et al. Prediabetes: a high-risk state for diabetes development. The Lancet 2012;379:2279-90.

2 Edwards CM, Cusi K. Prediabetes: a worldwide epidemic. Endocrinol Metab Clin North Am 2016;45:751-64.

3 Mahat RK, Singh N, Arora M, et al. Health risks and interventions in prediabetes: a review. Diabetes Metab Syndr 2019;13:2803-11.

4 Bansal N. Prediabetes diagnosis and treatment: a review. World $\mathrm{J}$ Diabetes 2015;6:296-303.

5 Saeedi P, Petersohn I, Salpea P, et al. Global and regional diabetes prevalence estimates for 2019 and projections for 2030 and 2045: Results from the International Diabetes Federation Diabetes Atlas, $9^{\text {th }}$ edition. Diabetes Res Clin Pract 2019;157:107843.

6 Spurr S, Bally J, Bullin C, et al. The prevalence of undiagnosed Prediabetes/type 2 diabetes, prehypertension/hypertension and obesity among ethnic groups of adolescents in Western Canada. BMC Pediatr 2020;20:31.

7 Mainous AG, Tanner RJ, Baker R, et al. Prevalence of prediabetes in England from 2003 to 2011: population-based, cross-sectional study. BMJ Open 2014;4:e005002.

8 Li G, Zhang P, Wang J, et al. The long-term effect of lifestyle interventions to prevent diabetes in the China dA Qing diabetes prevention study: a 20 -year follow-up study. The Lancet 2008;371:1783-9.

9 Lozano R, Naghavi M, Foreman K, et al. Global and regional mortality from 235 causes of death for 20 age groups in 1990 and 2010: a systematic analysis for the global burden of disease study 2010. The Lancet 2012;380:2095-128.

10 Cai X, Zhang Y, Li M, et al. Association between prediabetes and risk of all cause mortality and cardiovascular disease: updated metaanalysis. BMJ 2020;370:m2297.

11 Cai X, Liu X, Sun L, et al. Prediabetes and the risk of heart failure: a meta-analysis. Diabetes Obes Metab 2021;23:1746-53.

12 Erasmus RT, Blanco Blanco E, Okesina AB, et al. Prevalence of diabetes mellitus and impaired glucose tolerance in factory workers from Transkei, South Africa. S Afr Med J 2001;91:157-60.

13 Motala AA, Esterhuizen T, Gouws E, et al. Diabetes and other disorders of glycemia in a rural South African community: prevalence and associated risk factors. Diabetes Care 2008;31:1783-8.

14 Hird TR, Pirie FJ, Esterhuizen TM, et al. Burden of diabetes and first evidence for the utility of $\mathrm{HbA} 1 \mathrm{c}$ for diagnosis and detection of diabetes in urban black South Africans: the Durban diabetes study. PLoS One 2016;11:e0161966.

15 American Diabetes Association. Diagnosis and classification of diabetes mellitus. Diabetes Care 2014;37 Suppl 1:S81-90.

16 Kumar R, Nandhini LP, Kamalanathan S, et al. Evidence for current diagnostic criteria of diabetes mellitus. World J Diabetes 2016;7:396.

17 Hoy D, Brooks P, Woolf A, et al. Assessing risk of bias in prevalence studies: modification of an existing tool and evidence of interrater agreement. J Clin Epidemiol 2012;65:934-9.

18 Higgins JPT, Thompson SG. Quantifying heterogeneity in a metaanalysis. Stat Med 2002;21:1539-58.

19 Huedo-Medina TB, Sánchez-Meca J, Marín-Martínez F, et al. Assessing heterogeneity in meta-analysis: $Q$ statistic or $\mathrm{I}^{2}$ index? Psychol Methods 2006;11:193-206.

20 Guyatt GH, Oxman AD, Vist GE, et al. Grade: an emerging consensus on rating quality of evidence and strength of recommendations. $B M J$ 2008;336:924-6. 